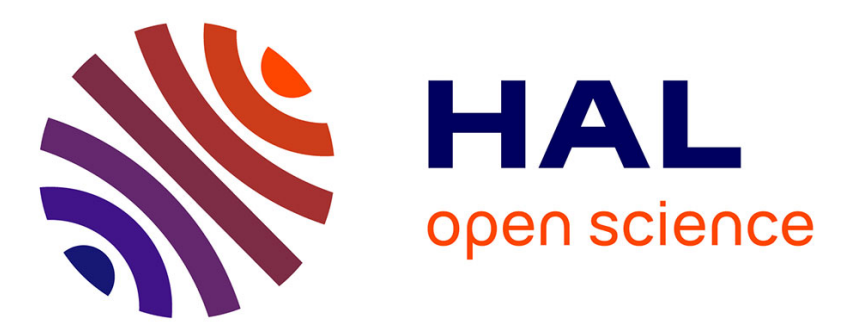

\title{
Assessment of the harmonic balance method on a self-oscillating one degree-of-freedom system with regularized friction
}

\author{
Pierre Vigué, Christophe Vergez, Sami Karkar, Bruno Cochelin
}

\section{To cite this version:}

Pierre Vigué, Christophe Vergez, Sami Karkar, Bruno Cochelin. Assessment of the harmonic balance method on a self-oscillating one degree-of-freedom system with regularized friction. Nonlinear Dynamics, 2018, 92 (3), pp.961-971. 10.1007/s11071-018-4102-8 . hal-01740425

\section{HAL Id: hal-01740425 \\ https://hal.science/hal-01740425}

Submitted on 22 Mar 2018

HAL is a multi-disciplinary open access archive for the deposit and dissemination of scientific research documents, whether they are published or not. The documents may come from teaching and research institutions in France or abroad, or from public or private research centers.
L'archive ouverte pluridisciplinaire HAL, est destinée au dépôt et à la diffusion de documents scientifiques de niveau recherche, publiés ou non, émanant des établissements d'enseignement et de recherche français ou étrangers, des laboratoires publics ou privés. 


\title{
Assessment of the harmonic balance method on a self-oscillating one degree-of-freedom system with regularized friction
}

\author{
Pierre Vigué ${ }^{a}$, Christophe $\operatorname{Vergez}^{a}$, Sami Karkar ${ }^{b}$, Bruno Cochelin $^{a}$ \\ a : Aix Marseille Univ, CNRS, Centrale Marseille, LMA, Marseille, France \\ b : Ecole Centrale de Lyon, 36 avenue Guy de Collongue, 69134 Ecully Cedex, France \\ \{ vigue, vergez \} @lma.cnrs-mrs.fr, sami.karkar@ec-lyon.fr, bruno.cochelin@centrale-marseille.fr
}

\begin{abstract}
Time-periodic solutions of dynamical systems can be looked for using a discretization method. This paper tests the Harmonic Balance Method (HBM) on a one-degree-of-freedom system (mass, damper, spring, belt) with a regularized friction law. Its relative error is computed with respect to the number of discretization unknowns. Despite the widespread idea that frequency methods are hardly applicable to friction problems, the HBM compares well with a classical time-domain method for this nonlinear system. The main conclusion of this article is that the HBM, without any specific optimization, is well suited for regularized friction.
\end{abstract}

\section{Introduction}

For nonlinear systems, the study of time-periodic solutions, and their evolution with respect to a parameter, is called the continuation of periodic solutions. For systems of Partial Differential Equations (PDE), continuation studies may follow two successive discretization methods: a spatial discretization to transform PDE into Ordinary Differential Equations (ODE), for instance, a modal projection; then a second procedure to transform ODE systems into algebraic systems. Thus, time discretization methods are an important ingredient in periodic solutions continuation, either in the time domain or in the frequency domain.

One of them is the harmonic balance method, where a periodic solution $\mathbf{x}$ of a nonlinear differential system

$$
\dot{\mathbf{x}}=\mathbf{f}(\mathbf{x}, \lambda)
$$

is sought after as:

$$
\mathbf{x}(t)=\mathbf{x}_{0}+\sum_{k=1}^{H} \mathbf{x}_{c, k} \cos (k \omega t)+\mathbf{x}_{s, k} \sin (k \omega t)
$$

The new unknowns are the Fourier coefficients $\left(\mathbf{x}_{0}, \mathbf{x}_{c, k}, \mathbf{x}_{s, k}\right)$ and $\omega$. One must compute the Fourier coefficients of $\mathbf{f}(\mathbf{x}, \lambda)$ given those of $\mathbf{x}$. First attempts, especially when carried out by hand, were limited to a few terms in the sum (2), that is, $H$ small (often, $H=1$ ). To overcome this issue the Alternating Frequency/Time Domain method (AFT) [11] computes the nonlinear terms in the time domain. Then, Cochelin and Vergez [4] showed that the computation of Fourier coefficients can be performed automatically for an arbitrary number of harmonics $H$, in the frequency domain, provided the differential system follows a specific formalism. Then, this can be coupled efficiently to a robust continuation technique, the Asymptotic Numerical Method (ANM) since the system obtained on Fourier coefficients follows the requirements of the ANM.

The main available results for a class of problems of a certain regularity are asymptotic convergence rates. As already underlined in [1], the lack of theoretical results on preasymptotic error analysis is problematic for applications and motivates numerical study. Indeed, convergence rates describe how methods behave when the number of discretization unknowns (for instance, the number of coefficients in Fourier series) tends to infinity. However, what are realistic error expectations for a given number of discretization unknowns? As recalled later, for $\mathbf{f}$ an analytic function in eq. (1), the HBM has a uniform convergence rate of $\mathcal{O}\left(c^{-H}\right)$, where $c>1$ and $H$ is the number of harmonics [5]. Although it is satisfying to have a convergence result, the 


\begin{tabular}{|l|l|l|}
\hline Symbol & Signification & Numerical value (if applicable) \\
\hline$\lambda$ & Continuation parameter & $\cdot$ \\
$V_{b}$ & Belt speed & $\cdot$ \\
$F_{N}$ & Normal force & $5 \mathrm{~N}$ \\
$\zeta_{1}$ & Damping & $1.3096 \times 10^{-3}$ \\
$\omega_{1}$ & Natural angular frequency & $1.2316 \times 10^{3} \mathrm{rad} . \mathrm{s}^{-1}$ \\
$M_{1}$ & Modal mass & $6.42 \times 10^{-3} \mathrm{~kg}$ \\
$\mu_{s}$ & Static friction coefficient & 0.4 \\
$\mu_{d}$ & Dynamic friction coefficient & 0.2 \\
$\varepsilon$ & Modulus smoothing parameter & $10^{-4}$ \\
$\alpha$ & Friction constant & $\alpha=\sqrt{\mu_{s}\left(\mu_{s}-\mu_{d}\right)} \simeq 0.283$ \\
$n$ & Regularization parameter & $\cdot$ \\
\hline
\end{tabular}

Table 1: Parameters used for the mass-spring-damper-belt system with regularized friction.

definition of this exponential convergence merely means that if $\mathbf{u}$ denotes the exact solution and $\mathbf{u}_{H}$ the $\mathrm{HBM}$ estimate with $H$ harmonics, there exists $c>1$, a rank $H_{0}$ and a constant $C_{0}$, such that

$$
\forall H>H_{0}, \quad\left\|\mathbf{u}_{H}-\mathbf{u}\right\|_{\infty}<C_{0} c^{-H}
$$

Obviously, the numbers $c, H_{0}$ and $C_{0}$ depend on the system of interest and are not known in advance. If $c$ is too close to unity the convergence will be rather slow; if $H_{0}$ is large the asymptotic behaviour will not be observed for practical (small) numbers $H$.

Another practical difficulty is the monotonicity of the error. If a solution is suspected to be inaccurate (for instance, it presents spurious oscillations), will the solution be improved by adding discretization unknowns? In order to compute approximate values of convergent series, increasing the number of terms in the partial sum is not always reliable. Although it may seem at this point a "worst case scenario", non-monotone error is a phenomenon encountered in the current study, and the authors wish to underline its importance.

The issues mentioned above stress the need for more theoretical results on preasymptotic error analysis. In the absence of such results, it is valuable to carry out numerical benchmarks. The case of regularized impact has been studied in [1]. Another numerically demanding interaction in nonlinear mechanics is friction, with a great variety of models developed for specific needs $[2,3]$. The present paper focuses on a regularized friction law. The solutions branches for this law and their similarities with Coulomb's friction law are examined in [6].

As a reference method, the Orthogonal Collocation at Gauss points with regular meshing is also used to solve for periodic solutions of this model since it is a widely used and accepted method for non linear or even piecewise-smooth systems. The aim of the current assessment is to evaluate the capacity of the HBM to deal with regularized friction. It is known that there are refinements which can be deployed and tried whenever necessary. The HBM can be more efficient using harmonic selection techniques (for instance, [7]). The OC can benefit from adaptive meshing (although it can also be defective, as mentioned in Section 5.3 of [8]). Our results show that, without optimization, on regularized friction, the error for the HBM has at least two interesting properties: it is relatively small; it is monotonically decreasing. In some cases, it can also reach its asymptotic rate with few unknowns.

The present paper is organised as follows: in section 2, model equations are given and numerical methods are recalled. Section 3 presents the protocol used to evaluate the numerical accuracy of the method. Section 4 presents the results of this error analysis, for two types of nonlinearity (weakly nonlinear or stiff, depending on the regularization parameter), and each time for two types of solutions.

\section{Model and theoretical background}

\subsection{Model equations}

The model studied in this paper is a mass-spring-damper-belt system (Fig. 1, a). This mechanical device is a bowed string toy model. If $y$ denotes the transverse displacement of a string at the bowing point $x=x_{b}$, 
a projection of the equation of motion on the first bending mode leads to a mass-spring-damper equation. Namely, if $V_{r}$ denotes the relative velocity between the mass and the belt, $V_{b}$ the belt velocity, $F_{N}$ the normal force, $M_{1}$ the modal string mass, $\zeta_{1}$ the damping, $\omega_{1}$ the natural angular frequency,

$$
\begin{gathered}
\ddot{y}+2 \zeta_{1} \omega_{1} \dot{y}+\omega_{1}^{2} y=\frac{F_{N}}{M_{1}} \mu_{n}\left(V_{r}\right) \\
\text { where } V_{r}=\dot{y}-V_{b}
\end{gathered}
$$

The contact law between the mass and the belt is a regularized friction law $\mu_{n}\left(V_{r}\right)$. It is based on an analytical mother function, $g$, and a regularization parameter, $n . g$ is defined as

$$
g\left(V_{r}\right):=\frac{-\mu_{d} V_{r} \sqrt{V_{r}^{2}+\varepsilon}-2 \alpha V_{r}}{V_{r}^{2}+1}, \quad \alpha=\sqrt{\mu_{s}\left(\mu_{s}-\mu_{d}\right)}
$$

where $\mu_{s}$ and $\mu_{d}$ are static and dynamic friction coefficients $\left(\mu_{s}>\mu_{d}\right)$. Then, $\mu_{n}$ is defined as

$$
\mu_{n}\left(V_{r}\right):=g\left(n V_{r}\right)=\frac{-\mu_{d} V_{r} \sqrt{V_{r}^{2}+\frac{\varepsilon}{n^{2}}}-2 \frac{\alpha}{n} V_{r}}{V_{r}^{2}+\frac{1}{n^{2}}}
$$

For small values of $n$ the system is weakly nonlinear; for great values of $n$ it becomes stiff. This regularized law $\mu_{n}$ is studied in [6]. A plot of the function $\mu_{n}$ is given in Fig. 1, b, for $n=10$ (weakly nonlinear system) and $n=100$ (stiff system). For this regularized law, the movement type is classified as follows: let $V_{n}$ be such that

$$
\mu_{n}\left(V_{n}\right)=\max _{v} \mu_{n}(v) \simeq \mu_{s}
$$

then

$$
\left|V_{r}(t)\right| \leqslant\left|V_{n}\right| \Rightarrow \text { stick, } \quad\left|V_{r}(t)\right|>\left|V_{n}\right| \Rightarrow \text { slip }
$$

A period containing exactly one interval of stick motion and one of slip motion is called stick-slip.

a)

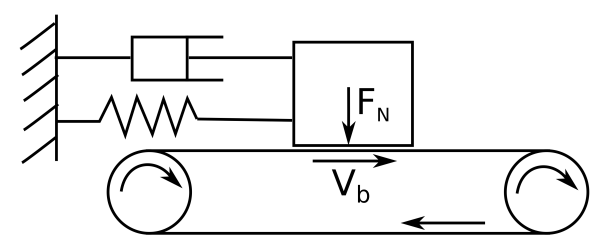

b)

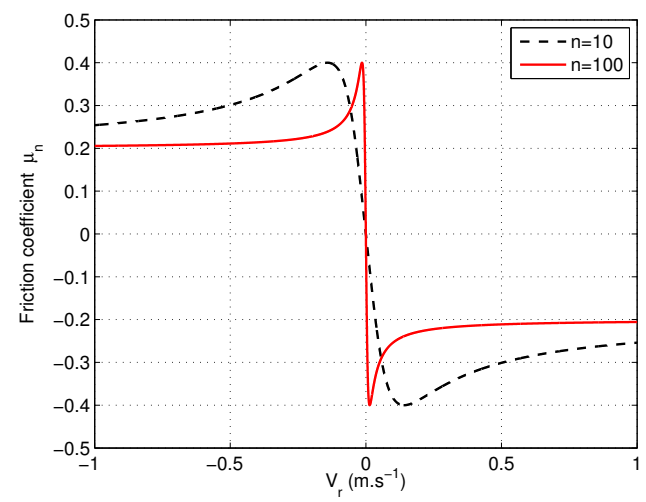

Figure 1: a) Mass-spring-damper system with a conveyor belt. b) Regularized friction law for $n=10$ (black, dash) and $n=100$ (red, solid).

\subsection{Numerical methods}

The association of the HBM and the Asymptotic Numerical Method (ANM), or of the OC and the ANM, is implemented in the Matlab toolbox MANLAB [9]. It requires what is called quadratic formulation. By adding auxiliary variables, the derivation order is lowered to 1 and the nonlinearities are recast into quadratic ones, so that $\mathbf{u}$, the vector of all unknown variables, is solution of

$$
\mathbf{m} \dot{\mathbf{u}}=\mathbf{c}_{0}+\lambda \mathbf{c}_{1}+\mathbf{l}_{0} \mathbf{u}+\lambda \mathbf{l}_{1} \mathbf{u}+\mathbf{q}_{0}(\mathbf{u}, \mathbf{u})
$$


where $\lambda$ is the continuation parameter, $\mathbf{m}, \mathbf{l}_{0}, \mathbf{l}_{1}$ are matrices, $\mathbf{c}_{0}, \mathbf{c}_{1}$ vectors, $\mathbf{q}_{0}$ a bilinear operator [4]. We recall here the quadratic formulation of equations $(3),(4),(6)$ chosen in [6]:

$$
\begin{array}{rlll}
0 & = & -\lambda & \\
0 & =\frac{\varepsilon}{n^{2}} & & \\
0 & =\frac{1}{n^{2}} & & -S \\
0 & = & & +2 \frac{\alpha}{n} V_{r} \\
\dot{y} & = & & +\omega_{1} z \\
\dot{z} & = & & -2 \zeta_{1} \omega_{1} z-\omega_{1} y+\frac{F_{N}}{\omega_{1} M_{1}} \mu_{n} \\
\mathbf{m} \dot{\mathbf{u}}=\mathbf{c}_{0} & +\lambda \mathbf{c}_{1} & & +\mathbf{l}_{0} \mathbf{u}
\end{array}
$$

The operators from eq. (9) are recalled ( $\mathbf{l}_{1}$ is null). The 6 variables are concatenated into a vector $\mathbf{u}=$ $\left(V_{r}, R, S, \mu_{n}, y, z\right)^{t}$.

We also recall briefly the two time-discretization methods, the HBM and the OC.

In the HBM, the periodic solution is sought after as a truncated Fourier series:

$$
\widehat{\mathbf{u}}(t)=\mathbf{u}_{0}+\sum_{k=1}^{H} \mathbf{u}_{c, k} \cos (k \omega t)+\sum_{k=1}^{H} \mathbf{u}_{s, k} \sin (k \omega t)
$$

New unknowns are vectors $\mathbf{u}_{0}, \mathbf{u}_{c, k}$ and $\mathbf{u}_{s, k}$, and angular frequency $\omega$. To approximate an analytic function, the HBM has a uniform asymptotic convergence rate of $\mathcal{O}\left(c^{-H}\right)$, where $c>1$ and $H$ is the number of harmonics [5]. The main difficulty in using the HBM with a large number of harmonics is to find Fourier coefficients of nonlinear terms. In our case that is the purpose of the quadratic formulation, as proposed in [4].

In the OC method, the solution is approximated by a periodic, continuous, piecewise polynomial of order $p$. The (unknown) period is divided into $N_{i n t}$ subintervals $\left[t_{i}, t_{i+1}\right]$. In each subinterval, equidistant time-steps $t_{i}^{j}$ and the samples of the solution $\mathbf{u}_{i}^{j}$ are defined as

$$
t_{i}^{j}:=t_{i}+\frac{j}{p} h, \quad \mathbf{u}_{i}^{j}:=\mathbf{u}\left(t_{i}^{j}\right), \quad 0 \leqslant j \leqslant p
$$

The approximate solution $\widehat{\mathbf{u}}$ is interpolated with $\mathcal{L}_{i}^{j}$, the Lagrange polynomials of order $p$ based on the timesteps $t_{i}^{j}$ :

$$
\forall t \in\left[t_{i}, t_{i+1}\right], \quad \widehat{\mathbf{u}}(t)=\sum_{j=0}^{p} \mathbf{u}_{i}^{j} \mathcal{L}_{i}^{j}(t)
$$

The solution is then assumed continuous and periodic, which gives constraints at $t=t_{i}$ and $t=0$. An algebraic system on the unknowns $\omega$ and vectors $\mathbf{u}_{i}^{j}$ is obtained by evaluation at the Gauss-Legendre points (i.e the zeros of the Legendre polynomial of order $p$ on each subinterval). Using regular meshing, the OC has a uniform asymptotic convergence rate of $\mathcal{O}\left(N_{\text {int }}^{-(p+1)}\right)$ [12]. As usual the convergence is obtained by increasing the number of subintervals, since it is cumbersome to increase the degree of polynomials (this would require computing zeroes of Legendre polynomials). From now on the polynomials order is set to $p=3$.

The number of discretization unknowns for each variable, $N_{\text {dis }}$, is defined as: $N_{\text {dis }}=2 H+1$, for the HBM, $N_{\text {dis }}=3 N_{\text {int }}+1$ for the OC.

\section{Comparison protocol}

\subsection{Reference solution}

Since both methods converge for $\mathcal{C}^{\infty}$ problems, we can use as a reference the solution given by one method with a great number of discretization unknowns. We use here the HBM with $H=500$ harmonics. Numerical 
integration, though it would provide an independent reference, has accuracy limitations and preliminary tests showed that it was not always reliable. For instance, for $n$ sufficiently large, in a portion of the bifurcation diagram near the first Hopf bifurcation, where the amplitude and the angular frequency of the solution are steep functions of the continuation parameter $V_{b}$ (see [6]), usual numerical integration schemes seem unable to compute periodic solutions.

\subsection{Norm}

Two kinds of results about numerical error are displayed below. First, one can focus on each variable separately by computing relative errors. A comparison between the six variables can then be carried out. However, this comparison is meaningful only if all variables have the same (null) mean value. One can notice for instance that equation (10a) brings an offset between $z$ and $V_{r}$, which would yield different relative errors. Therefore, for each variable $X$ in the vector $\mathbf{u}$, its mean value is removed before computing the relative error.

For each variable $X$, after evaluating $X_{\text {ref }}$ and $X_{\text {test }}$ in $N_{t}=10^{5}$ points regularly spaced over their respective periods, which creates two vectors $\left(X_{\text {ref }}\left(t_{j}\right)\right)_{j},\left(X_{\text {test }}\left(t_{j}\right)\right)_{j}$, its mean value $M(X)$ is defined by

$$
M(X):=\frac{1}{N_{t}} \sum_{k \geqslant 0} X_{\mathrm{ref}}\left(t_{k}\right)
$$

The relative error without bias is defined by

$$
\epsilon_{r}(X):=\frac{\left\|\left(X_{\mathrm{ref}}\left(t_{j}\right)\right)_{j}-\left(X_{\mathrm{test}}\left(t_{j}\right)\right)_{j}\right\|_{2}}{\left\|\left(X_{\mathrm{ref}}\left(t_{j}\right)\right)_{j}-M(X)\right\|_{2}}
$$

The second kind of results is scalar and uses a single norm. It has already been highlighted that using the ANM makes necessary the introduction of 4 auxiliary variables, namely $V_{r}, R, S$, $\mu_{n}$, instead of using just 2 , $y$ and $z$, as a numerical integration method would. For the sake of a complete assessment of the error, the second norm takes the 6 variables into account. Thus, with $\mathbf{u}=\left(V_{r}, R, S, \mu_{n}, y, z\right)^{t}$, vector of the 6 variables, $\left(\mathbf{u}^{i}\left(t_{j}\right)\right)_{1 \leqslant i \leqslant 6,1 \leqslant j \leqslant N_{t}}$ the matrix of its time evaluations, $M_{\mathbf{u}}$ the mean values of each variable, mean relative error $\epsilon_{r, m}$ is now defined as

$$
\epsilon_{r, m}:=\frac{\left\|\left(\mathbf{u}_{\mathrm{ref}}^{i}\left(t_{j}\right)\right)_{i, j}-\left(\mathbf{u}_{\mathrm{test}}^{i}\left(t_{j}\right)\right)_{i, j}\right\|_{2}}{\left\|\left(\mathbf{u}_{\mathrm{ref}}^{i}\left(t_{j}\right)\right)_{i, j}-M_{\mathbf{u}}\right\|_{2}}
$$

\subsection{Test solution}

When the number of discretization unknowns (i.e. $H$ or $N_{\text {int }}$ ) changes, the solution branch is altered. For instance, for the system (3), the fold bifurcation (see Fig. 2), located at $V_{b} \simeq 10.26 \mathrm{~m} . \mathrm{s}^{-1}$ (for $n=10$ ), is displaced to $V_{b} \simeq 24.05 \mathrm{~m} . \mathrm{s}^{-1}$ for $H=1, V_{b} \simeq 7.51 \mathrm{~m} . \mathrm{s}^{-1}$ for $H=5$ and $V_{b} \simeq 9.46 \mathrm{~m} . \mathrm{s}^{-1}$ for $H=9$. To compare two solutions, a reference on the one hand, and a test solution with less harmonics or less subintervals on the other hand, a criterion is needed to perform a relevant comparison. As in a previous comparison protocol [1], an energy criterion is chosen. In the test solution, the mass needs to have the same kinetic energy (over a period) as in the reference.

At last, branches of solutions are available. Where should tests be performed, i.e. which solutions should be chosen as references? To the author's knowledge, the number of unknowns needed to approximate a solution cannot be estimated in advance. One can also observe that this number can vary along a solution branch. For the system considered here, near the two Hopf bifurcations (see Fig. 2) and on the slipping portion of the branch, the solution requires few unknowns and does not present any numerical challenge. Between the first Hopf bifurcation and the belt velocity denoted $V_{1}$ on the one hand, and between the velocity $V_{2}$ and the fold bifurcation on the other hand, the solution is a (regularized) stick-slip with $V_{r}$ staying negative over a period. Between the velocities $V_{1}$ and $V_{2}, V_{r}$ briefly changes sign, which, as the authors found in preliminary tests, makes this stick-slip more challenging to compute. Also, the stiffness of the system, which is controlled by the regularization parameter $n$, alters the solution, and is a relevant parameter of interest. Therefore, four cases will be studied: 


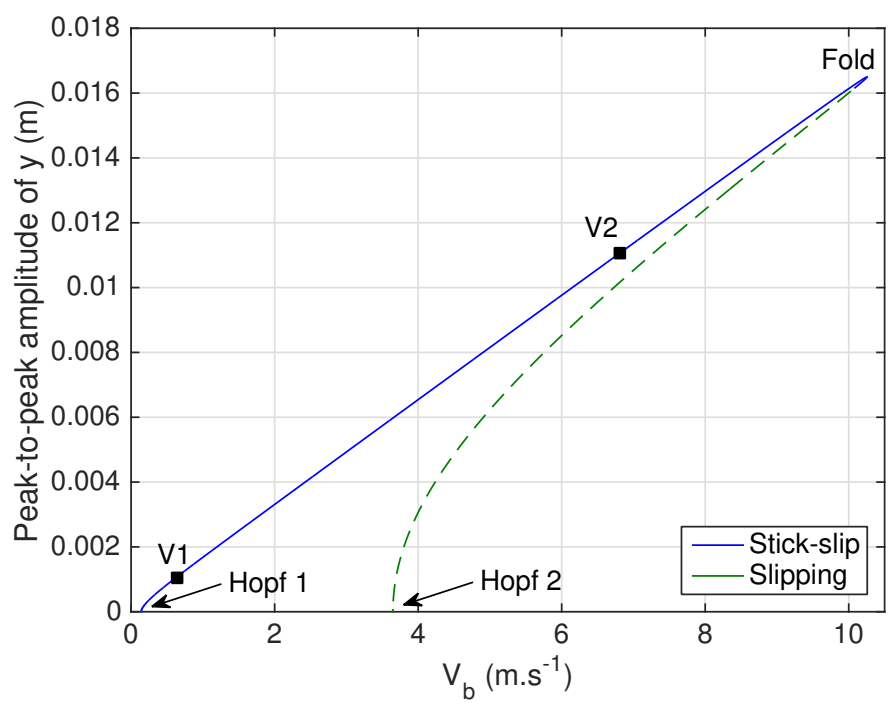

Figure 2: Bifurcation diagram for the system (3), $n=10$ : peak-to-peak amplitude of $y$ vs $\lambda=V_{b}$ (HBM, $H=80$ ). Solid line: stable solution, dash: unstable.

1. "Weakly nonlinear system, situation 1", denoted W1: low value of $n(n=10), V_{r}$ stays negative (section 4.1)

2. "Weakly nonlinear system, situation 2", denoted W2: low value of $n(n=10), V_{r}$ briefly changes sign (section 4.2)

3. "Stiff system, situation 1", denoted S1: high value of $n(n=100), V_{r}$ stays negative (section 4.3)

4. "Stiff system, situation 2", denoted S2: high value of $n(n=100), V_{r}$ briefly changes sign (section 4.4).

\section{Error results}

\subsection{Case W1: weakly nonlinear system, situation 1}

a)

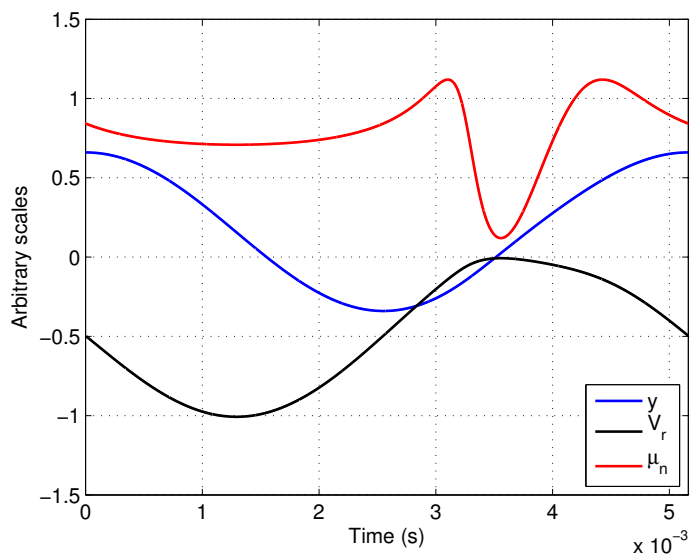

b)

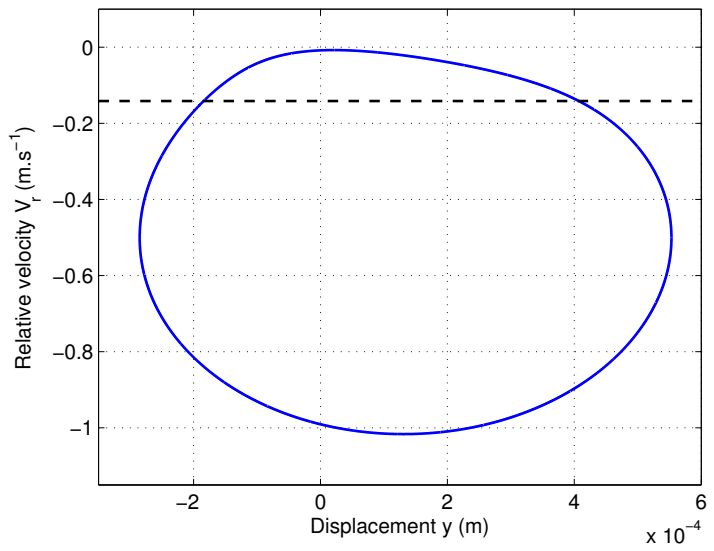

Figure 3: Reference solution (computed with HBM, $H=500$ ), in Case W1 (as defined in section 4.1) $: n=10, V_{b}=0.5 \mathrm{~m} . \mathrm{s}^{-1}$ other parameters values are given in Table 1. a) One period of the displacement $y$, relative velocity $V_{r}$ and friction coefficient $\mu_{n}$ with arbitrary vertical scales. b) Phase diagram $\left(y, V_{r}\right)$ in blue, limit velocity between stick and slip $V_{n}$ in black dashed (see equation (8)). 
a)

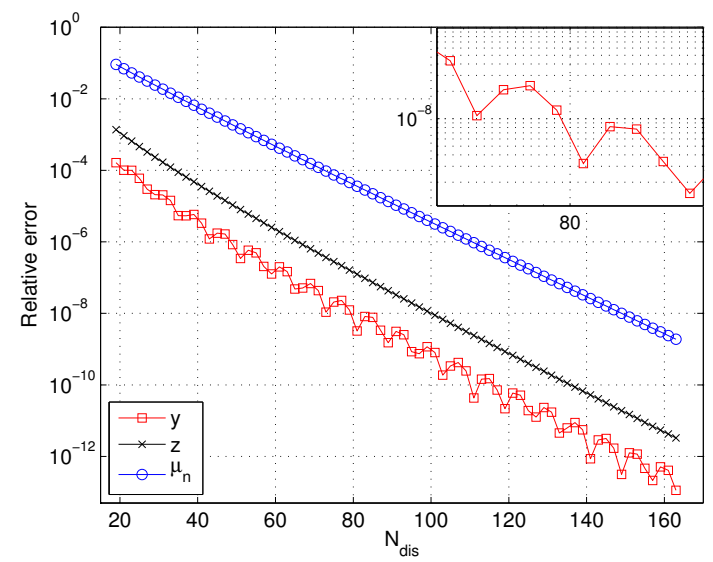

b)

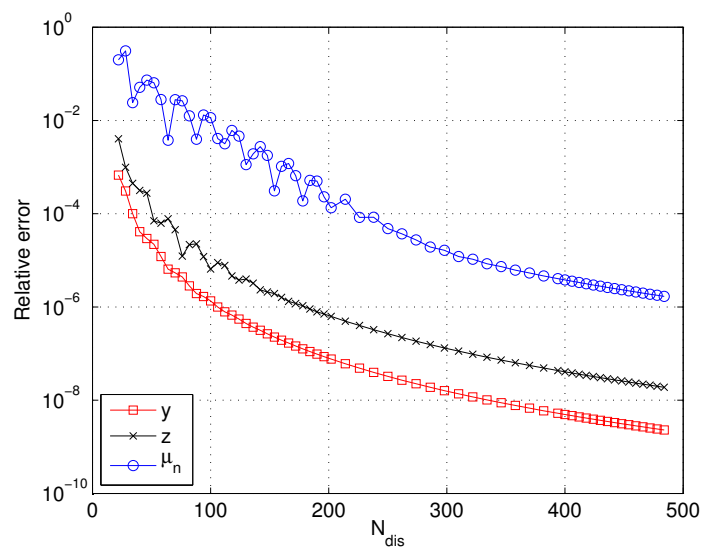

Figure 4: Relative errors (eq. 15) for three specific variables $y, z$ and $\mu_{n}$, in case W1 (as defined in section 4.1). a) HBM, semilog scale, with a zoom window on $y$ around $N_{\text {dis }}=80$. b) OC, semilog scale. Note that axis limits are different between the two figures.

a)

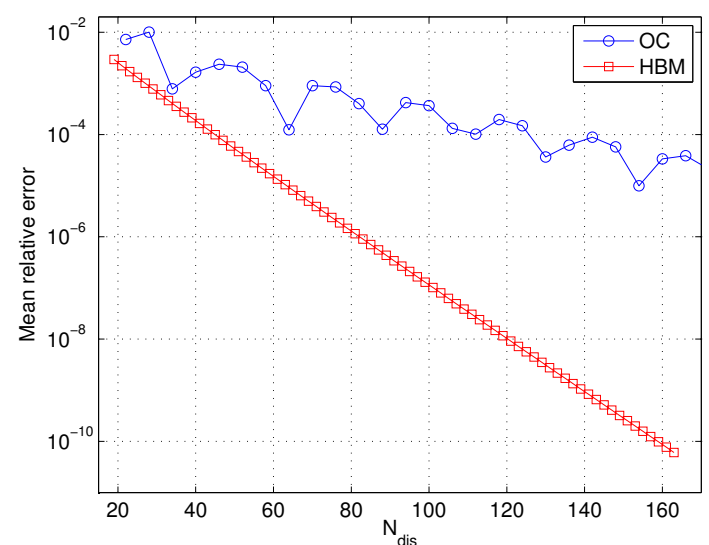

b)

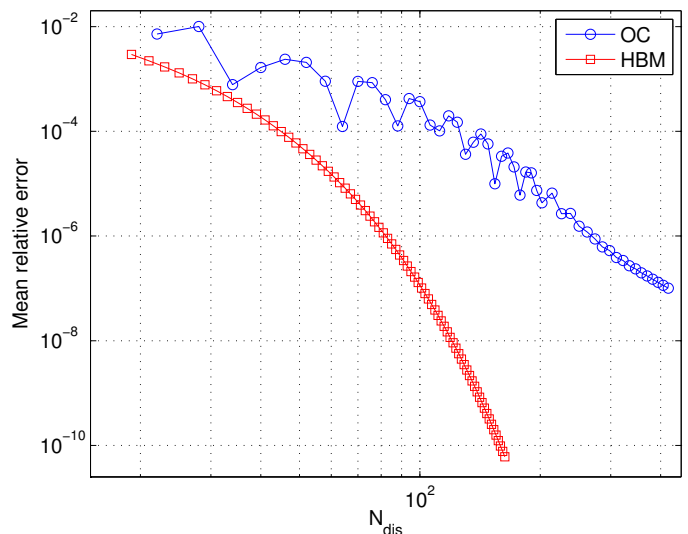

Figure 5: Mean relative error (eq. 16) for the HBM and the OC, in case W1. a) Semilog scale. b) Log-log scale. 
For a low value of the regularization parameter $(n=10)$, which means, for a weakly nonlinear system, the case $\mathrm{W} 1$ focuses on a reference solution with a small belt velocity $\left(V_{b}=0.5 \mathrm{~m} . \mathrm{s}^{-1}\right)$. For this first choice of $n$ and $V_{b}$, Fig. 3, left, shows one period of the displacement $y$, relative velocity $V_{r}$ friction coefficient $\mu_{n}$. Fig. 3, right, shows the cycle in the phase space $\left(y, V_{r}\right)$. An important characteristic of this solution is that $V_{r}$ is negative during the period, unlike the solution presented in case W2 (section 4.2).

For any number of discretization unknowns $N_{\text {dis }}, y$ is more accurately computed than $z$, which is more precise than $\mu_{n}$ (Fig. 4). When the friction law is Coulomb's law, defined as:

$$
\mu\left(V_{r}\right)= \begin{cases}-\mu_{d} \operatorname{sign}\left(V_{r}\right) & \text { if } V_{r} \neq 0 \text { (slip) } \\ \mu_{0} \text { with }\left|\mu_{0}\right| \leqslant \mu_{s}, & \text { if } V_{r}=0, \text { (stick) }\end{cases}
$$

then during a stick-slip cycle, the acceleration and the friction coefficient have a discontinuity between sticking and slipping. The velocity is continuous with two singular points over a period, and differentiable elsewhere. Although the regularization process makes every variable analytical with respect to the continuation parameter, the regularized variables $y, z$ and $\mu_{n}$ have different spectral content. This can explain a better accuracy for some variables compared to others, even if they are all smooth.

For $z$ and $\mu_{n}$ the errors for the HBM are straight lines in the semilog plot (in agreement with the asymptotic convergence rate $\mathcal{O}\left(c^{-H}\right), c>1$ ) for small values of $N_{\text {dis }}$. The variable $y$ is very accurately represented with the HBM (more than the five others); but from $N_{\text {dis }}=43$ to 163 , small ripples appear very regularly (as displayed in the zoom window around $N_{\text {dis }}=80$ ) and the ripples span 3 or 4 steps. This intriguing behaviour does not undermine the precision of this method since it is a small effect on the most accurately computed variable.

The OC exhibits up to two stages in the preasymptotic range for each variable (Fig. 4b). At first, the error can be a non-monotone function of $N_{\text {dis }}$. Then, the error is strictly decreasing, and its slope coefficient in $\log$-log scale converges to its expected value $-(p+1)=-4$ for an asymptotic convergence rate of $\mathcal{O}\left(N_{\text {int }}^{-(p+1)}\right)$.

The transition between these two stages depends on the stiffness of the variables. The error is immediately a monotone function of $N_{\text {dis }}$ for $y$; it becomes monotone for $z$ when $N_{\text {dis }} \geqslant 130$; for $\mu_{n}$, this happens when $N_{\text {dis }} \geqslant 238$.

In terms of mean relative error, the HBM shows a uniform convergence rate $\mathcal{O}\left(c^{-H}\right)$, which means a linear fit in semilog scale (Fig. 5, left). For $61 \leqslant N_{\text {dis }} \leqslant 183$ the linear coefficient is $-0.052 ;$ since $N_{\text {dis }}=2 H+1$, $c=\exp (2 * 0.052 * \ln (10))=1.27$.

The asymptotic polynomial convergence of the OC appears later. At first sight there is an almost straight line in log-log scale from $N_{\text {dis }} \geqslant 250$, but the slope coefficient may be different from the asymptotic rate when the error starts being monotone. A linear regression fits the curve with coefficient -4.3 for $394 \leqslant N_{\text {dis }} \leqslant 484$.

The accuracy of the chosen reference solution can be checked a posteriori. Indeed, for a variable $X$, let $X_{e}$ be the exact solution, $X_{\text {test }}$ and $X_{\text {ref }}$ being the test and the reference as above. Then:

- $\epsilon_{1}=\left\|X_{\text {test }}-X_{e}\right\|$ is the error that is searched for ;

- $\epsilon_{2}=\left\|X_{\text {ref }}-X_{e}\right\|$ has to be a small error ;

- $\epsilon_{3}=\left\|X_{\text {test }}-X_{\text {ref }}\right\|$ is actually measured.

Reverse triangle inequality gives $\left|\epsilon_{1}-\epsilon_{3}\right| \leqslant \epsilon_{2}$ and means that as long as $\epsilon_{3}$ is much greater than $\epsilon_{2}, \epsilon_{3}$ is close to $\epsilon_{1}$. If the HBM mean error is still a straight line (in semilog scale) when $N_{\text {dis }}$ increases, the test solution is still much less accurate than the reference, and the assumption of a small error $\epsilon_{2}$ is correct. On the contrary, if machine precision prevents the error $\epsilon_{2}$ from being small (compared with $\epsilon_{3}$ ), the test and the reference become close. In that case, from a certain $N_{\text {dis }}$, there is a plateau, with an almost constant error beyond this number of unknowns. This plateau, not shown in figures, is not an issue of the convergence process, but one of its features: it indicates when the test reaches the same accuracy as the reference. For the HBM and $n=10$, this plateau appears beyond $N_{\text {dis }}=200$ for $y, N_{\text {dis }}=220$ for $z$, and $N_{\text {dis }}=260$ for $\mu_{n}$.

A comparison with numerical integration over the variables $y$ and $z$ was used to confirm our results. Let's call ODE the solution provided by the the solver ode15s from Matlab ODE suite [13]. Since the relative error between ODE and the HBM reference solution (with $H=500$ harmonics) is about $10^{-11}$ (resp. $10^{-10}$ ) for $y$ (resp. $z$ ), choosing ODE instead of HBM as a reference gives extremely close error curves for those variables. 
a)

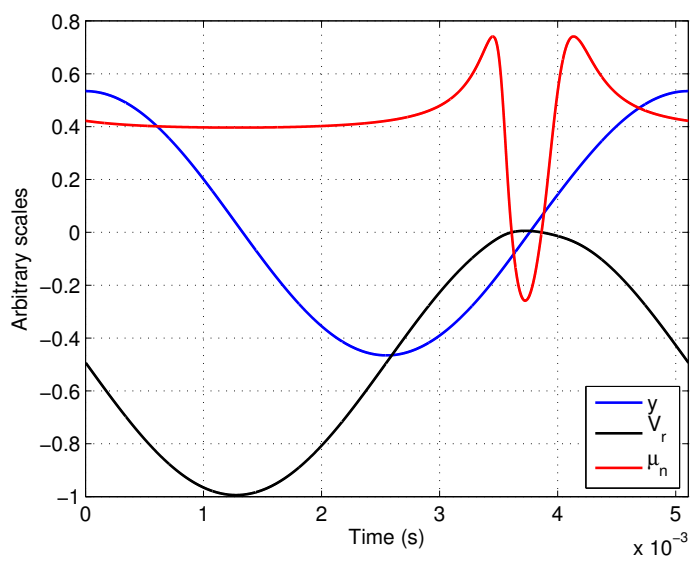

b)

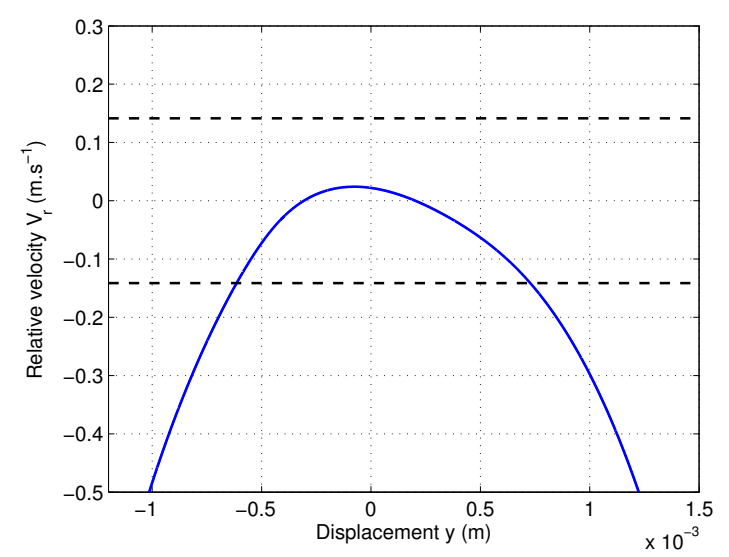

Figure 6: Reference solution (computed with HBM, $H=500$ ), in Case W2 (as defined in section 4.2) : $n=10$, $V_{b}=2$ m.s ${ }^{-1}$, other parameters values are given in Table 1. a) One period of the displacement $y$, relative velocity $V_{r}$ and friction coefficient $\mu_{n}$, with arbitrary vertical scales. b) Zoom in the phase diagram $\left(y, V_{r}\right)$ in blue ; limit velocities between stick and slip, $V_{n}$ and $-V_{n}$, are black dashed lines (see equation (8)).

\subsection{Case W2: weakly nonlinear system, situation 2}

For the same value of the regularization parameter $(n=10)$, the case W2 focuses on a reference solution with a greater belt velocity $\left(V_{b}=2 \mathrm{~m} . \mathrm{s}^{-1}\right)$. The reference solution is plotted in Fig. 6 . On the right, phase diagram $\left(y, V_{r}\right)$ is zoomed during the stick motion $\left(\left|V_{r}\right| \leqslant V_{n}\right)$. The sign of $V_{r}$ changes during a period. It is the main difference with the previous case W1. This property of the solution has significant consequences on the preasymptotic error (as shown below), but the underlying reasons are still unclear.

Note that as underlined in [6], this sign change is a difference with the stick-slip cycles obtained using Coulomb's law, where the relative velocity has a constant sign. It is caused by the regularization process and has no physical interpretation.

In this case (Fig. 7), the HBM error decreases at a fast rate for small values of $N_{\text {dis }}$, then reaches a slower asymptotic convergence (straight lines in the semilog plot). Similarly to the first case, the relative error on $y$ is smaller than the one on $z$, itself smaller than the error on $\mu_{n}$. Ripples on the error curves now affect the three variables, not only $y$.

The OC error curves exhibit spurious oscillations, especially for the variables $y$ and $\mu_{n}$ until large values of $N_{\text {dis }}$. This time, the six error curves are presented on the same figure to highlight that for small number of unknowns, the error curves are close for the two methods. The computation of mean errors (Fig. 8) gives a synthetic measure, where the error on $\mu_{n}$ appears predominantly.

\subsection{Case S1: stiff system, situation 1}

For increasing values of $n$, the system becomes stiffer (Fig. 1, b). The chosen value here is $n=100$, and in this first situation $\mathrm{S} 1$, similarly to case $\mathrm{W} 1, V_{r}$ stays negative over one period (for the reference solution $\left.V_{b}=0.05 \mathrm{~m} \cdot \mathrm{s}^{-1}\right)$. The relative errors over $y, z$ and $\mu_{n}$ are ordered as before: $y$ has the smallest relative error, $\mu_{n}$ the largest. The two methods have closer error curves than before (Fig. 10). The HBM is already exponentially convergent, with monotone errors on $z$ and $\mu_{n}$ (and small ripples on $y$ ). The OC mean error is non-monotone in the tested range (Fig. 10). Going further should show the asymptotic convergence of the OC mean error, as observed for $n=10$ in case W1.

The stiffness of the system, compared with case W1 $(n=10)$, is highlighted by the number of discretization unknowns needed to reach a specific accuracy. Let's consider the variable $y$. In case W1, the error was inferior to $10^{-4}$ when $N_{\text {dis }} \geqslant 21$ for the HBM, when $N_{\text {dis }} \geqslant 34$ for the OC. When $n=100$ in this analogous case S1, more discretization unknowns are needed to keep the error on $y$ lower than $10^{-4}\left(N_{\text {dis }} \geqslant 97\right.$ for the HBM, $N_{\text {dis }} \geqslant 148$ for the OC). 


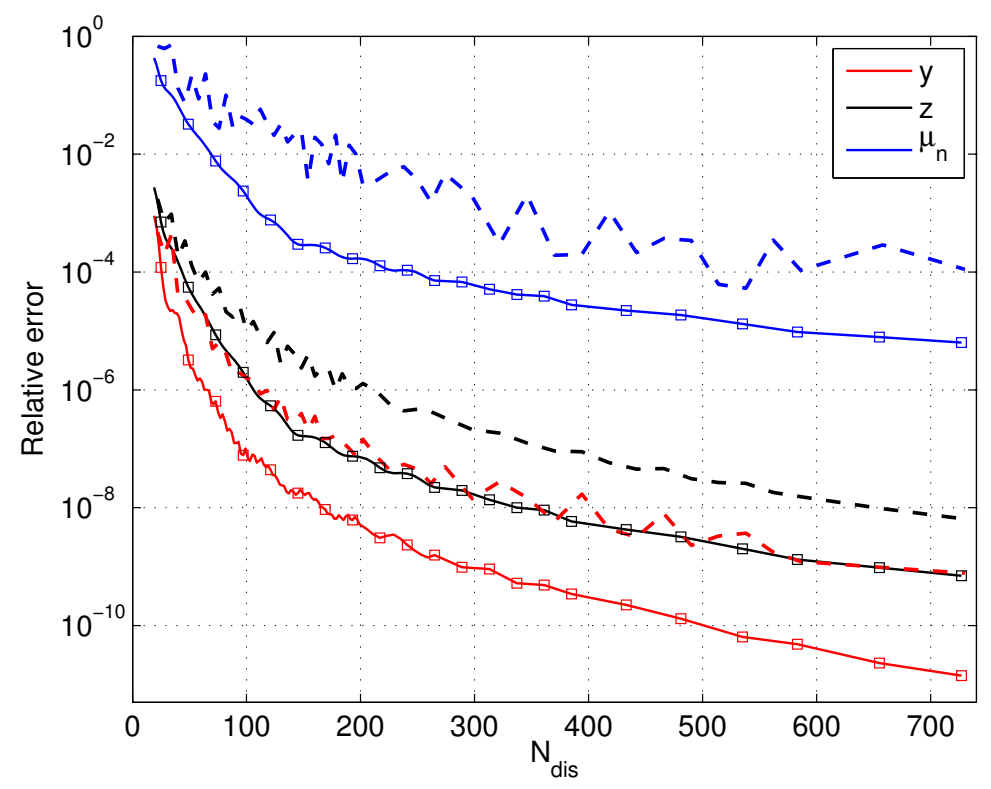

Figure 7: Relative errors (eq. (15)) for three specific variables $y, z$ and $\mu_{n}$. Weakly nonlinear system $(n=10)$, case W2 (as defined in section 4.2). Solid lines with squares: HBM, dashed lines: OC. Semilog scale.

a)

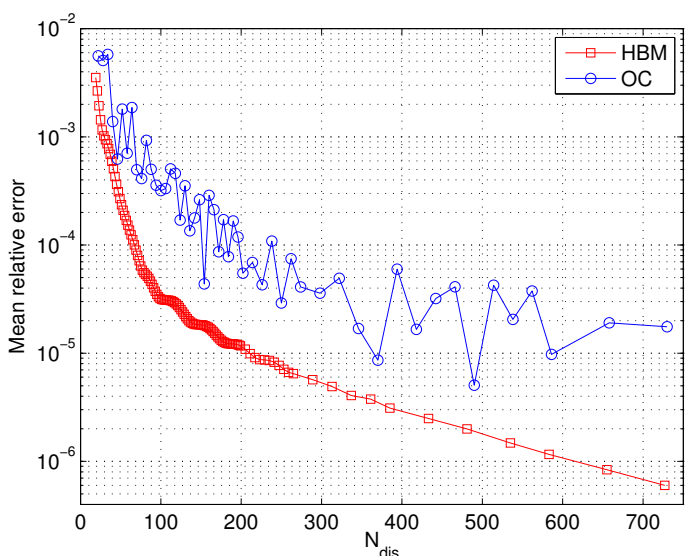

b)

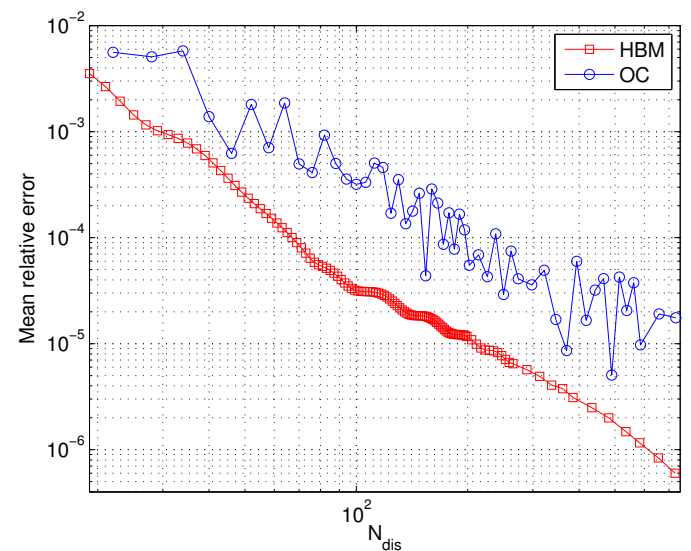

Figure 8: Mean relative error (eq. (16)) for the HBM and the OC, for a weakly nonlinear system $(n=10)$ in case W2 (as defined in section 4.2). a) Semilog scale. b) Log-log scale. 


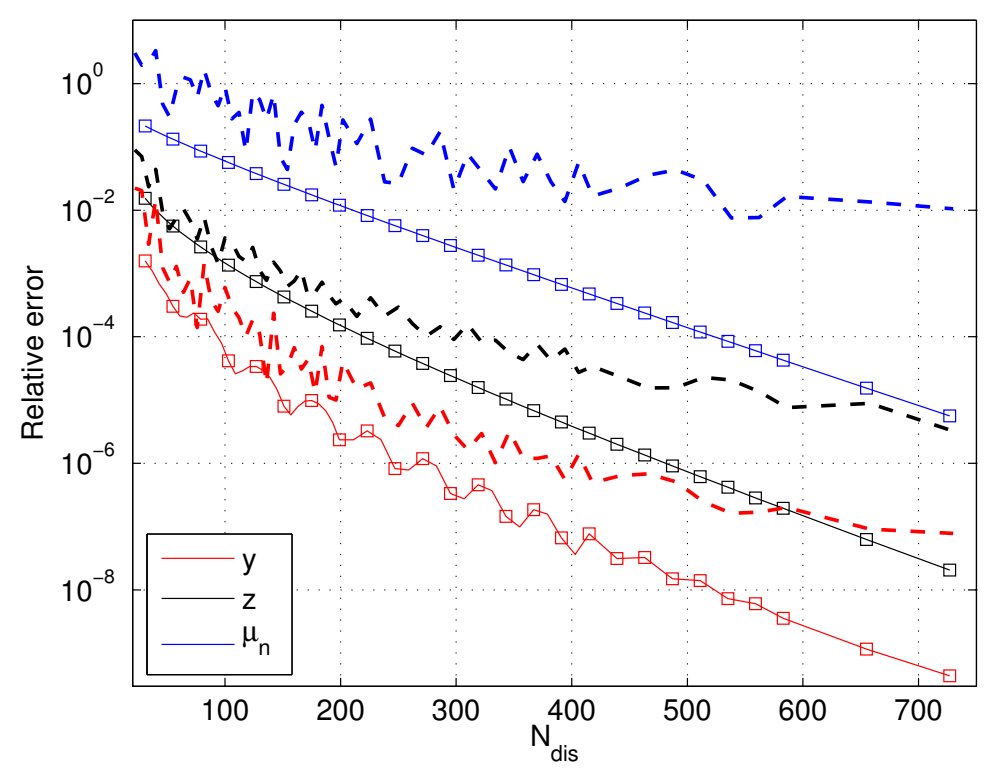

Figure 9: Relative errors (eq. 15) for three specific variables $y, z$ and $\mu_{n}$. Case S1 (as defined in section 4.3). Solid lines with squares: HBM, dashed lines: OC. Semilog scale.

a)

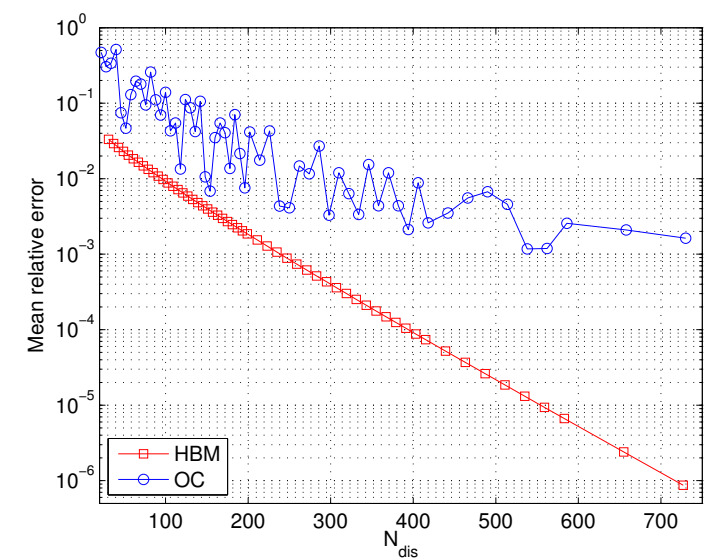

b)

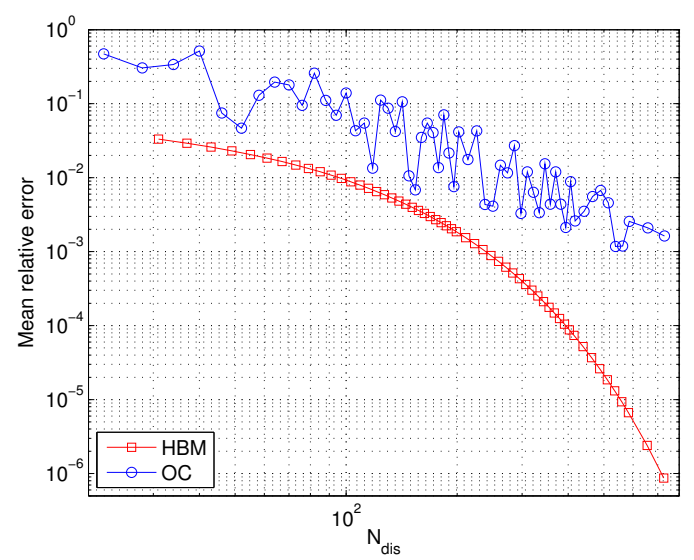

Figure 10: Mean relative error (eq. (16)), for the HBM and the OC, for case S1 (as defined in section 4.3). a) Semilog scale. b) Log-log scale. 


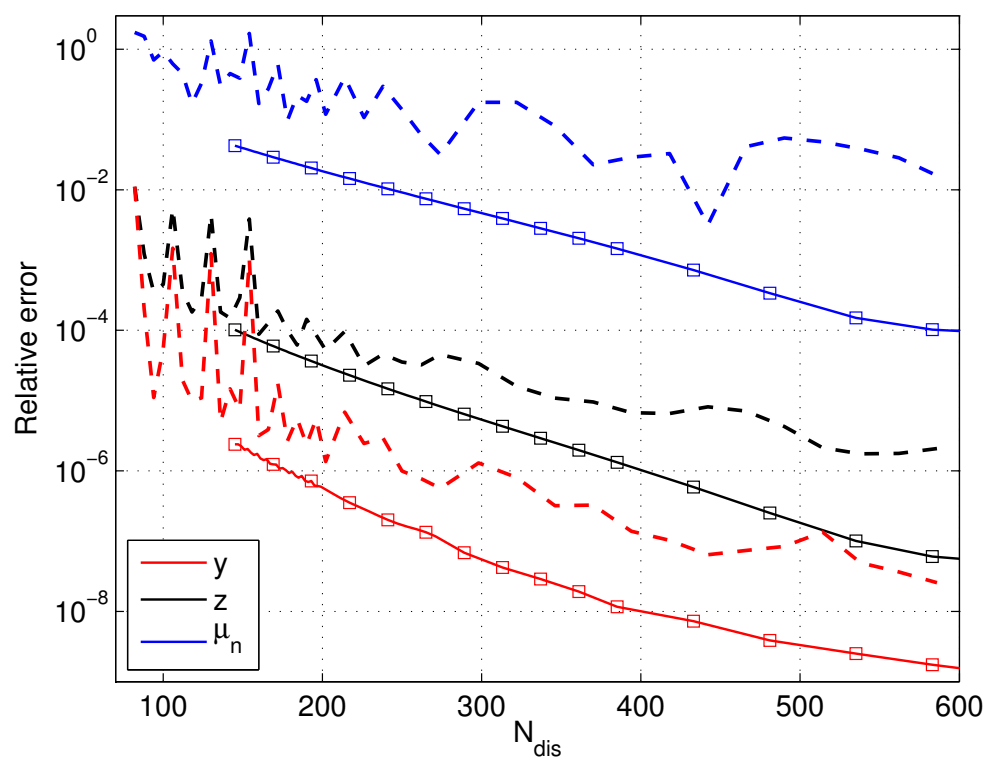

Figure 11: Relative errors (eq. (15)) for three specific variables $y, z$ and $\mu_{n}$, for case S2 (defined in Section 4.4). Solid lines with squares: HBM, dashed lines: OC. Semilog scale.

\subsection{Case S2: stiff system, situation 2}

In this last case S2, the system is stiff $(n=100)$; and for the reference solution, the value of $V_{b}=0.4$ $\mathrm{m} . \mathrm{s}^{-1}$ is chosen so that the sign of $V_{r}$ changes shortly during the period (analogous of W2, section 4.2). The errors on the three variables $y, z$ and $\mu_{n}$ are shown in Fig. 11. The HBM converges with monotone errors. The rates slow down for large number of discretization unknowns. The OC errors exhibit important spikes for small $N_{\text {dis }}$, and errors are non-monotone in the tested range. Once more, mean error curves (not displayed here) only reflect the predominant error on $\mu_{n}$. In Fig. 11 the HBM error curves start at $N_{\text {dis }}=145$ and the OC curves start at $N_{\text {dis }}=82$. If $N_{\text {dis }}$ is too small, the HBM cannot be used to continue the whole branch. On the contrary, the $\mathrm{OC}$ is able to continue the branch but computed solutions can be inaccurate.

\section{Conclusion}

In this paper, the Harmonic Balance Method (HBM) is assessed on a nonlinear system with a regularized friction law. A numerical evaluation of the error with respect to the number of discretization unknowns is performed for four different cases (either a weakly or highly nonlinear system ; either a constant sign for the relative velocity or a varying sign). The Orthogonal Collocation at Gauss points with regular meshing (OC) is used to put results into context.

In cases where the relative velocity keeps a constant sign, the HBM immediately starts converging at an asymptotic rate. That rate is slower for a stiff system than for a weakly nonlinear system. The OC presents non-monotone errors for small numbers of discretization unknowns, and the asymptotic convergence of the OC can be difficult to observe when the system is stiff.

In cases where the sign of the relative velocity shortly changes over a period, the HBM does not converge at its asymptotic rate from the beginning, and the OC errors exhibit more important oscillations. The HBM presents satisfyingly low error values. We conclude that the HBM, without any specific optimization, is well suited for regularized friction.

\section{Acknowledgements}

This work has been carried out in the framework of the Labex MEC (ANR-10-LABX-0092). The project leading to this publication has received funding from Excellence Initiative of Aix-Marseille University - 
A*MIDEX, a French "Investissements d'Avenir" programme.

\section{References}

[1] S. Karkar, C. Vergez, B. Cochelin: A comparative study of the harmonic balance method and the orthogonal collocation method on stiff nonlinear systems. Journal of Sound and Vibration, 333, 12, p. 2554-2567, 2014.

[2] B. Armstrong-Hélouvry, P. Dupont, C. Canudas de Wit: A survey of models, analysis tools and compensation methods for the control of machines with friction. Automatica 30 (7), p. 1083-1138, 1994.

[3] J. Awrejcewicz, P. Olejni: Analysis of dynamic systems with various friction laws. ASME Applied Mechanics Review 58, p. 389-411, 2005.

[4] B. Cochelin, C. Vergez: A high order purely frequency-based harmonic balance formulation for continuation of periodic solutions. Journal of Sound and Vibration, 324, p. 243-262, 2009.

[5] E. Tadmor. The exponential accuracy of Fourier and Chebyshev differencing methods. SIAM Journal on Numerical Analysis, 23 (1), p. 1-10, 1986.

[6] P. Vigué, C. Vergez, S. Karkar, B. Cochelin: Regularized friction and continuation: comparison with Coulomb's law. Journal of Sound and Vibration, 389, p. 350-363, 2017.

[7] A. Grolet, F. Thouverez: On a new harmonic selection technique for harmonic balance method. Mechanical Systems and Signal Processing, 30, p. 43-60, 2012.

[8] K. Engelborghs, T. Luzyanina, K.I.T. Hout, D. Roose: Collocation methods for the computation of periodic solutions of delay differential equations. SIAM Journal on Scientific Computing, 22 (5), p. 1593$1609,2001$.

[9] S. Karkar, R. Arquier, B. Cochelin, C. Vergez, A. Lazarus, O. Thomas, MANLAB, An Interactive Continuation Software. URL <http://manlab.lma.cnrs-mrs.fr> (accessed December 2017)

[10] D. Gottlieb, S. A. Orszag. Numerical Analysis of Spectral Methods : Theory and Applications, SIAM, Philadelphia, Pennsylvania, 1977.

[11] T. M. Cameron, J. H. Griffin. An Alternating Frequency/Time Domain Method for Calculating the Steady-State Response of Nonlinear Dynamic Systems, Journal of Applied Mechanics, 56, p. 149-154, 1989.

[12] C. de Boor, B. Swartz. Collocation at Gaussian points, SIAM Journal of Numerical Analysis, 10, p. 582$606,1973$.

[13] L.F. Shampine, M.W. Reichelt, The Matlab ODE suite, SIAM Journal on Scientific Computing, 18, p. 1-22, 1997.

[14] K. Engelborghs, T. Luzyanina, D. Roose. Numerical bifurcation analysis of delay differential equations using DDE-Biftool, ACM Transactions on Mathematical Software, 28 (1), p. 1-21, 2002. 